Proc. of 12th International Workshop on Positron and Positronium Chemistry, August 28-September 1, 2017, Lublin, Poland

\title{
Effect of Ultraviolet Absorber on Photo-Degradation of Epoxy Coating Studied by Slow Positron Beam
}

\author{
Zheng Wang ${ }^{a}$, Fuwei LiU ${ }^{a}$, Jinguing Li ${ }^{a}$, Chunqing He ${ }^{a}$, Xiangyang Peng ${ }^{b}$, \\ Zhen HuAnG ${ }^{b}$ AND Pengfei FAnG ${ }^{a, *}$ \\ ${ }^{a}$ Department of Physics and Hubei Nuclear-Solid Physics Key Laboratory, Wuhan University, Wuhan 430072, China \\ ${ }^{b}$ Electric Power Research Institute of Guangdong Power Grid Co., Ltd, Guangzhou 510080, China
}

\begin{abstract}
The photo-degradation progress of epoxy coating and the effect of ultraviolet absorber under UV-B irradiation have been investigated in slow positron beam and by the Fourier transform infrared spectrometer. After $120 \mathrm{~h}$ of irradiation, the value of $S$ parameter in sample bulk is reduced while compared with the virgin sample. The result is mostly due to post-cure process happening in this initial irradiation stage. As the irradiation time increases to $360 \mathrm{~h}$, the $S$ parameter decreases sharply. This is due to the growth of carbonyl group and the generation of free radical. After $528 \mathrm{~h}$ or longer time of irradiation, a very low $S$ value was obtained near sample surface, indicating the formation of a dead surface layer. Positron results also reveal that the addition of ultraviolet absorber suppresses the development of the dead layer after long-term UV-B irradiation. Ultraviolet absorber has a suppressing effect on generation of polar groups towards sample bulk. The addition of ultraviolet absorber is a key factor that affects the photo-degradation of epoxy coating.
\end{abstract}

DOI: 10.12693/APhysPolA.132.1523

PACS/topics: epoxy coating, photo-degradation, ultraviolet absorber, slow positron beam, microstructure

\section{Introduction}

Epoxy resins are widely used as long-term protective coatings in various industrial applications. Durability is a primary concern for this protective coating system. The service lifetime of epoxy coating can be affected by its running environment, such as ultraviolet (UV) light, weather, moisture and salt. Because most polymers in coating systems absorb UV light [1], the UV irradiation becomes one of the most principal ageing factors. Thus, many attempts have been made to investigate the characterization of epoxy resins during UV degradation.

The effects of the radiation wavelength on the photoageing progress of epoxy resin are studied by Rivaton et al. $[2,3]$. They have found that the long wavelength radiation induces formation of phenyl structures, while the short wavelength exposure does not. Liu and coworkers [4] studied the microstructure evolution of epoxy coating during UV irradiation using electrochemical impedance spectroscopy. Monney et al. $[5,6]$ also investigated the photochemical degradation of an anhydride-epoxy system using different techniques. From now on, at least to our knowledge, few studies exist now which adequately cover the effect of ultraviolet absorber on depth profile degradation behavior of epoxy coating during UV irradiation.

Usually, the deterioration of a material structure spreads from the polymer surface to the bulk during the ageing process. Traditional characterization techniques, like X-ray photoelectron spectroscopy (XPS) and scan-

*corresponding author; e-mail: fangpf@whu.edu.cn ning electron microscope (SEM) can only provide information of surface structure. Extended study of the deteriorating surface structure including its depth profile information is an urgent requirement. In this paper, we report the UV ageing results of epoxy coating using an innovative technique, positron annihilation spectroscopy (PAS) coupled with a slow positron beam, which can provide depth profile information of a sample by adjusting the emitted positron energy [7]. Meanwhile, the chemical structure changes were evaluated by attenuated total reflectance Fourier transform infrared spectrometer (ATRFTIR). The effects of ultraviolet absorber on epoxy coating photo-degradation process were also discussed.

\section{Experimental}

\subsection{Materials and preparation}

Diglycidyl ether of bisphenol-A epoxy resin (DGEBA) E51 (Jiangsu San Group Corporation, China) and low molecular polyamide resin (LMPAR) 651 (Yueyang Zhongzhan Science \& Technology Co., Ltd.) were used for this study. The epoxy value of DGEBA is about $0.51 \mathrm{~mol} / 100 \mathrm{~g}$. Cross-linked DGEBA/LMPAR coating system was prepared by mixing DGEBA with recommended dosage of LMPAR. The UV absorber, 2-hydroxy4-methoxybenzophenone (UV-531), was added into the coating system at a weight ratio of $0.5 \%$, then continuous stirring for $0.5 \mathrm{~h}$ at room temperature. The samples were applied on carbon steel (the steel with carbon content up to $2.1 \%$ by weight) substrates and then cured in an oven at $80^{\circ} \mathrm{C}$ for $3 \mathrm{~h}$. The thickness of the dry films was $30 \pm 2 \mu \mathrm{m}$.

The ultraviolet (UV) irradiation of polyamide-cured DGEBA was performed in a UV weathering test chamber (XR-UV, Shenzhen Xiangrui Testing Instrument Co., 
Ltd. China) using commercial UV-B lamps (center wavelength $313 \mathrm{~nm}$ ). The UV radiation power density of $55 \mathrm{~mW} / \mathrm{cm}^{2}$ was obtained. Distance between UV the lamps and a sample was $7.0 \mathrm{~cm}$. The ageing temperature was regulated at $60^{\circ} \mathrm{C}$. All the samples were exposed to UV radiation with different extended periods of time: 0 , $120,360,528,864 \mathrm{~h}$, respectively.

\subsection{Characterization}

Chemical structure changes of a sample were studied by an attenuated total reflectance Fourier transform infrared spectrometer (ATR-FTIR, Nicolet iS10). Data acquisition used a resolution of $4 \mathrm{~cm}^{-1}$ and data spacing of $1.929 \mathrm{~cm}^{-1}$; each spectrum was an average of 32 scans, taken from 550 to $4000 \mathrm{~cm}^{-1}$.

A conventional line shape parameter, $S$, obtained with energy variable Doppler broadening energy spectroscopy (DBES, Wuhan University) was employed to characterize the ageing behavior of epoxy under UV-B irradiation. The DBES spectra were recovered at room temperature $\left(23^{\circ} \mathrm{C}\right)$ as a function of positron energy from 0.25 to $26 \mathrm{keV}$. Positrons were generated with a $1.85 \mathrm{GBq}$ of ${ }^{22} \mathrm{Na}$, moderated by a thin layer of solid $\mathrm{Ne}$, and then electromagnetically transported to the samples. The DBES spectra were recorded using a high-purity Ge solidstate detector (EG\&G Ortec) with an energy resolution of $1.3 \mathrm{keV}$ at the $511 \mathrm{keV}$ peak. The total count of each data point is $2 \times 10^{6}$ with a counting rate of $1200 \mathrm{cps}$. The positron annihilation line shape $S$ parameters from DBES were calculated as the ratio of the central area $(510.24 \mathrm{keV}<\mathrm{E}<511.76 \mathrm{keV})$ to the total area of the positron annihilation $\gamma$ peak after background subtraction.

\section{Results and discussion}

\subsection{ATR-FTIR analysis}

The ATR-FTIR spectra of the unmixed epoxy coating system obtained before and after UV ageing for 120 , 360, 528, $864 \mathrm{~h}$ are represented in Fig. 1a. The peak at $1639 \mathrm{~cm}^{-1}$, corresponding to $-\mathrm{C}=\mathrm{O}$ in amide group of polyamide, moves to wave number of $1664 \mathrm{~cm}^{-1}$ and is broadened. Meanwhile, the $\mathrm{C}-\mathrm{H}$ bond in methylene group near 2923 and $2852 \mathrm{~cm}^{-1}$ decreases. The result suggests a new amide is generated as a result of the oxidization of $\mathrm{C}-\mathrm{H}$ bond in $\alpha$-position of nitrogen atom on amino groups $[8,9]$. However, for the sample with ultraviolet absorber, the peak of $-\mathrm{C}=\mathrm{O}$ in amide group moves from $1639 \mathrm{~cm}^{-1}$ to $1656 \mathrm{~cm}^{-1}$, which is narrower than the unmixed one (from $1639 \mathrm{~cm}^{-1}$ to $1664 \mathrm{~cm}^{-1}$ ), indicating a suppressed effect of ultraviolet absorber on generation of the new amide group. As shown in Fig. 1b, because of the adding of ultraviolet absorber (UV-531), there is an inconspicuous increase in $1729 \mathrm{~cm}^{-1}$ for the 0 h-irradiated sample, and this peak is ascribed to the $-\mathrm{C}=\mathrm{O}$ in $\mathrm{UV}-531$. After $\mathrm{UV}$ irradiation, this peak is covered by a new peak generated in $1715 \mathrm{~cm}^{-1}$, which is corresponding to $-\mathrm{C}=\mathrm{O}$ in saturated aldehyde, ketone, or acid [4], suggesting the generation of $-\mathrm{C}=\mathrm{O}$ as a result of UV irradiation.
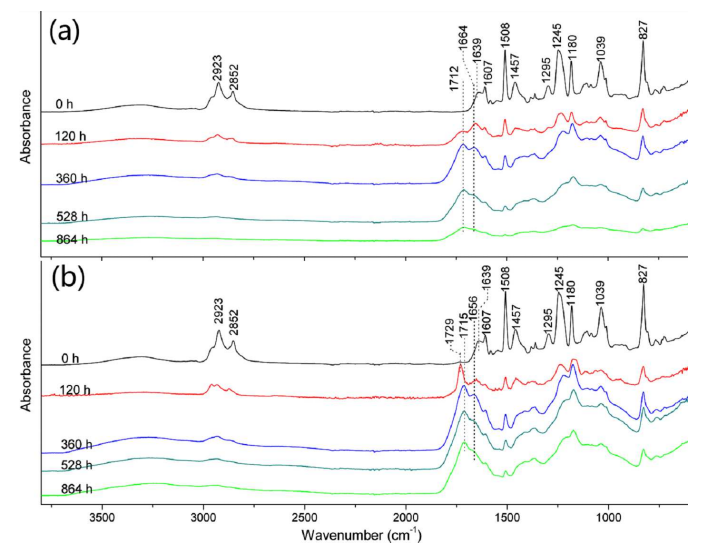

Fig. 1. ATR-FTIR spectra of unmixed epoxy coating (a) and 0.5 wt\% ultraviolet absorber (UV-531) containing epoxy coating (b) before and after UV-B radiation for different time.

Usually, the benzene ring is stable and does not participate in the degradation reaction during UV irradiation. Then the $-\mathrm{C}=\mathrm{C}$ in benzene ring, appearing at $1508 \mathrm{~cm}^{-1}$ in Fig. 1, is chosen as the reference peak. The generation of $-\mathrm{C}=\mathrm{O}$ in epoxy coating affects its microstructure characteristics and the anticorrosion properties [4], so this group is chosen for study. The ratio of areas of the $-\mathrm{C}=\mathrm{O}$ peak (appearing at $1712 \mathrm{~cm}^{-1}$ in Fig. $1 \mathrm{a}$, and $1715 \mathrm{~cm}^{-1}$ in Fig. 1b) and the $-\mathrm{C}=\mathrm{C}$ peak of benzene ring is defined as: $S^{\prime}=\frac{P A_{-\mathrm{C}=\mathrm{O}}}{P A_{-\mathrm{C}=\mathrm{C}}}$, and $\mathrm{PA}$ represents the peak area in ATR-FTIR spectrum. The variation of $S^{\prime}$ as a function of UV irradiation time is shown in Fig. 2 and the error bar is obtained by measuring three different points on sample surface. During the early stage of UV irradiation $(\leq 528 \mathrm{~h})$, the $S^{\prime}$ value of epoxy/UV531 sample is higher than the unmixed one, which could be contributed by the carbonyl group in ultraviolet absorber. However, after 864 h of irradiation, the $S^{\prime}$ value of epoxy/UV531 sample becomes smaller than the unmixed sample. The result indicates the $\mathrm{UV}$ absorber had a suppressed effect on the generation of $-\mathrm{C}=\mathrm{O}$ during $\mathrm{UV}$ irradiation.

\section{2. $S$ parameter analysis}

The $S$ parameters of unmixed epoxy coating before and after different periods of UV-B irradiation as a function of incident positron energy $E$ are shown in Fig. 3. The mean implantation depth of the positron as a result of inelastic interactions with polymer molecules is expressed in Eq. (1) [10], which is shown on the top horizontal axis of Fig. 3:

$$
z_{m}=\left(40 \times 10^{3} / \rho\right) E_{+}^{1.6},
$$

where $z_{m}$ is the mean implantation depth $(\mathrm{nm}), \rho$ is the density of the material $\left(\mathrm{kg} / \mathrm{m}^{3}\right)$, and $E_{+}$is the incident energy $(\mathrm{keV})$. In this study, the density of the epoxy system composites is around $1.16 \mathrm{~g} / \mathrm{cm}^{3}$.

As shown in Fig. 3, the $S$ value of non-irradiated sample increases with increasing incident energy until $2 \mathrm{keV}$. Similar to most polymers, this is ascribed to the back scattering and back diffusion of the positrons and positro- 


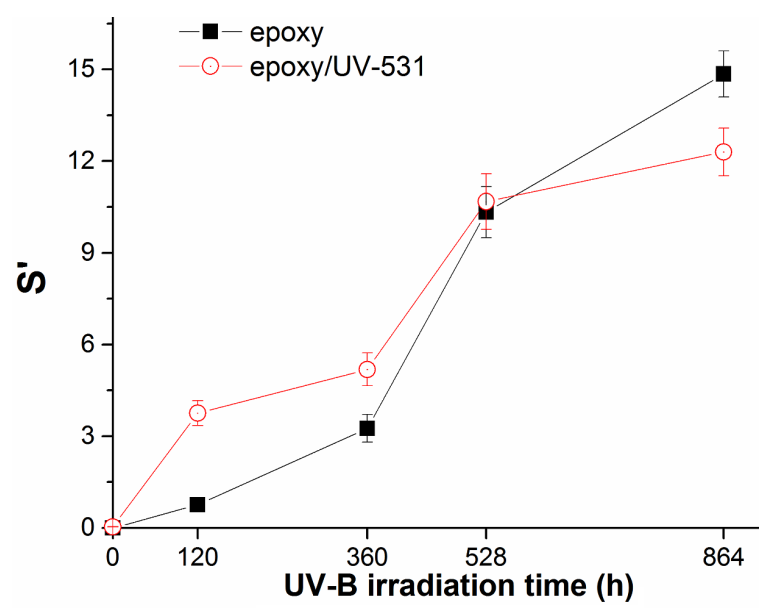

Fig. 2. Variation of $S^{\prime}$ value for epoxy coating with and without the addition of $0.5 \mathrm{wt} \%$ of ultraviolet absorber (UV-531) after UV-B radiation for different time.

nium (Ps) from or near the sample surface [11]. The formation possibility of $p$-Ps decreases, thus resulting in a low $S$ parameter. As the positron incident energy is larger than $2 \mathrm{keV}, S$ parameter value reaches a constant value, indicating the penetration of positron into the bulk of the coating.

After 120 h of irradiation, the variation trend of $S$ parameter is very similar to that of the virgin sample. However, compared to the non-irradiated one, the values of the $S$ parameter are lower over the whole incident energy range. The change in chemical environment influences the Ps formation and thus affects the value of $S$ parameter. In Fig. 1a, a new peak around $1712 \mathrm{~cm}^{-1}$, corresponding to $-\mathrm{C}=\mathrm{O}$, is formed after $120 \mathrm{~h}$ of UV irradiation. Polar groups can inhibit the formation of Ps and thus make the $S$ parameter decrease [12-14]. In addition, during the initial stage of degradation, there exists a post curing process in the network of epoxy coating, which leads to a better crosslinked structure $[15,16]$. The reduction of free volume can also induce the decrease of $S$ parameter.

After $360 \mathrm{~h}$ of UV irradiation, $S$ parameter decreases dramatically near the coating surface, shown in Fig. 3. However, the $S$ parameter in the sample bulk remains the same with the 120 h-irradiated one, which indicates the degradation concentrates on the sample surface, in a depth of about $2 \mu \mathrm{m}$. On one hand, more $-\mathrm{C}=\mathrm{O}$ is generated at this irradiation time, shown in Fig. 1a. The growth of the polar groups can reduce the $S$ parameter in large scale. On the other hand, UV irradiation induces chain scission reactions on coating surface, resulting in the formation of hydroxyl radicals [17]. Residual radicals, which are not consumed by oxygen in air, will reduce the formation of Ps, then contributing to a lower $S$ parameter.

After $528 \mathrm{~h}$ of irradiation, the $S$ parameter is low and remains unchanged with increasing incident energy $(\leq 6 \mathrm{keV})$ near sample surface. This region has been identified as dead surface layer after UV irradiation [1]. With increasing irradiation time, polar groups are gradually generated towards sample bulk. Thus, low $S$ parameter near sample surface remains unchanged. Besides, a new type of surface layer with a highly cross-linked structure may be formed by recombination of the generated radicals during UV irradiation. The reduction of free volume also results in a lower $S$ parameter near sample surface.

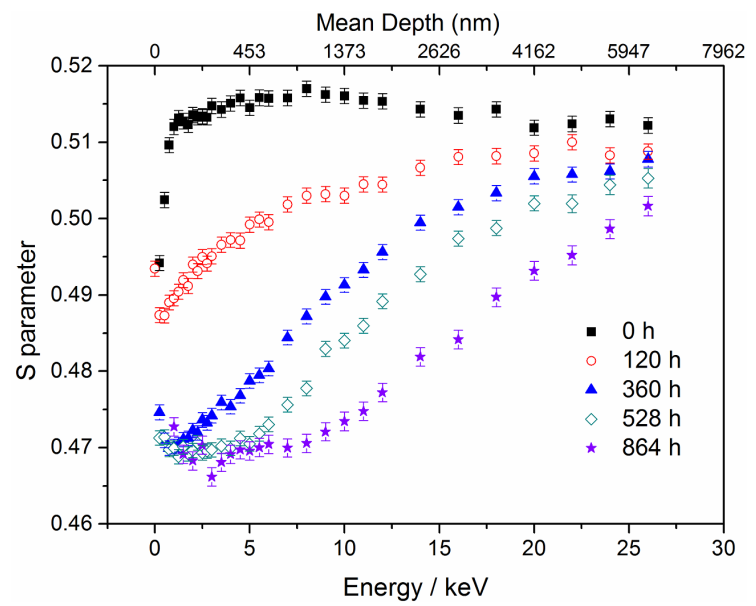

Fig. 3. $S$ parameter versus $E$ for unmixed epoxy coating exposed to UV-B irradiation for different time.

The effects of the ultraviolet absorber on the process of UV degradation were also investigated. Figure 4 shows the variation of $S$ parameter versus $E$ for $0.5 \mathrm{wt} \%$ of ultraviolet absorber containing epoxy coating after different time of UV irradiation. During the early stage of UV irradiation ( $\leq 528 \mathrm{~h}$ ), the $S$ parameter variation of epoxy/UV531 sample is similar to the unmixed one. Whereas, it takes $360 \mathrm{~h}$ of irradiation for epoxy/UV531 sample to form a dead surface layer, which is earlier than the unmixed sample $(528 \mathrm{~h})$. The result could be ascribed to the carbonyl group in ultraviolet absorber. However, during the long term of irradiation $(>528 \mathrm{~h}$ ), the surface dead layer of epoxy/UV531 sample does not grow with UV irradiation time, which indicates a lower growing rate of polar groups on sample surface. As shown in Fig. 4, the $S$ parameter trend of the 864 h-irradiated sample is about the same to the 528 h-irradiated one. The result suggests the addition of ultraviolet absorber suppresses the long-term degradation process on coating surface.

In $S(E)$ curve, the position where $S$ parameter begins to achieve a relative constant value is usually determined by sample surface condition. Figure 5 shows the variation of this injection depth after different time of UV irradiation for the unmixed sample and epoxy/UV531 sample. For the $120 \mathrm{~h}$ irradiated group, the injection depth where $S$ parameter reaches a constant of epoxy/UV531 sample is extremely lower than the unmixed sample. This can be attributed to the effect of UV-531, which can transfer UV light energy into heat, thus the epoxy/UV531 sample surface is much better cured than the unmixed one. 


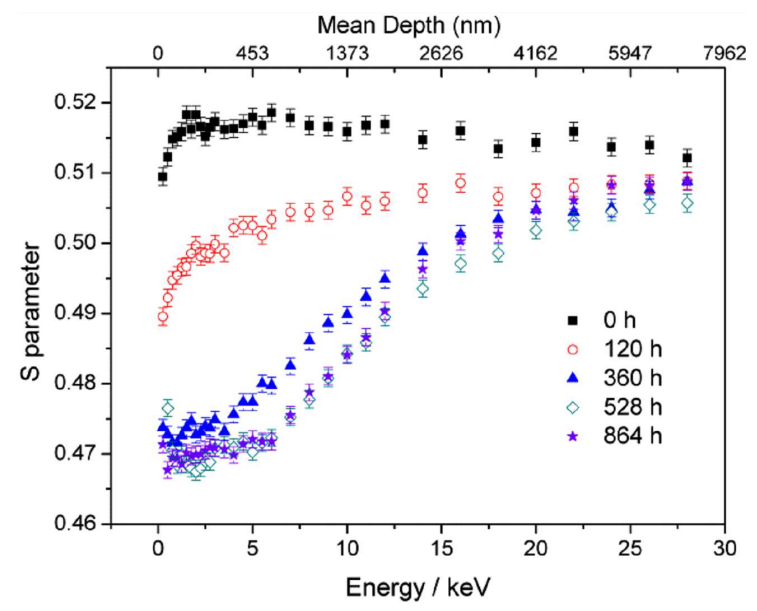

Fig. 4. $S$ parameter versus $E$ for $0.5 \mathrm{wt} \%$ of ultraviolet absorber (UV-531) containing epoxy coating exposed to UV-B irradiation for different time.

After 360,528 , or $864 \mathrm{~h}$ of irradiation, the degradation of epoxy/UV531 sample concentrates on its surface, indicating the suppressing effect of UV-531 on generation of polar groups towards sample bulk. Thus, during longer time irradiation, the depth where the $S$ parameter reaches a constant value is always lower for the epoxy/UV531 sample than for the unmixed one. All these results obtained from ATR-FTIR or slow positron beam indicate that the addition of ultraviolet absorber into epoxy coating makes its performance during the UV irradiation better.

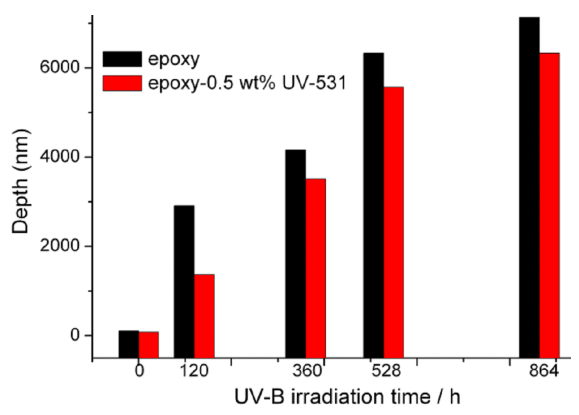

Fig. 5. Variation of the injection depth of positron corresponding to emission energy at which $S$ parameter begins to achieve a relative constant value as the increase of the irradiation time before and after the addition of ultraviolet absorber.

\section{Conclusion}

Slow positron beam was applied to study the photodegradation behaviors of epoxy-polyamide system during UV-B irradiation. During the initial stage of irradiation, there mainly happens a post-cure process in coating network. This makes the $S$ parameter in sample bulk reduced. With increasing irradiation time, carbonyl groups and radicals are generated and thus again reduce the value of the $S$ parameter. Moreover, a dead layer near sample surface is also formed, which is associated with a high concentration of polar groups and hydroxyl radicals as well as highly cross-linked structures. The addition of ultraviolet absorber suppresses the development of the dead layer after long-term UV-B irradiation. Ultraviolet absorber suppresses generation of polar groups towards sample bulk, and makes the degradation of epoxy coating concentrated on its surface.

\section{Acknowledgments}

This work was financially supported by the National Natural Science Foundation of China (No. 21174108), the Science and Technology Project of China Southern Power Grid Co., Ltd. (K-GD2014-185) and the Largescale Instrument and Equipment Sharing Foundation of Wuhan University (No. LF20170857).

\section{References}

[1] R. Zhang, X. Gu, H. Chen, J. Zhang, Y. Li, T. Nguyen, T.C. Sandreczki, Y.C. Jean, J. Polym. Sci. B Polym. Phys. 42, 2441 (2004).

[2] A. Rivaton, L. Moreau, J.-L. Gardette, Polym. Degrad. Stab. 58, 333 (1997).

[3] A. Rivaton, L. Moreau, J.-L. Gardette, Polym. Degrad. Stab. 58, 321 (1997).

[4] F. Liu, M. Yin, B. Xiong, F. Zheng, W. Mao, Z. Chen, C. He, X. Zhao, P. Fang, Electrochim. Acta 133, 283 (2014).

[5] L. Monney, R. Belali, J. Vebrel, C. Dubois, A. Chambaudet, Polym. Degrad. Stab. 62, 353 (1998).

[6] L. Monney, N. Rouge, C. Dubois, A. Chambaudet, Polym. Degrad. Stab. 62, 367 (1998).

[7] Y.C. Jean, P.E. Mallon, R. Zhang, H. Chen, Y. Li, J. Zhang, Y.C. Wu, T.C. Sandreczki, R. Suzuki, T. Ohdaira, X. Gu, T. Nguyen, Radiat. Phys. Chem. 68, 395 (2003).

[8] P. Musto, G. Ragosta, P. Russo, L. Mascia, Macromol. Chem. Phys. 202, 3445 (2001).

[9] B. Dao, J. Hodgkin, J. Krstina, J. Mardel, W. Tian, J. Appl. Polym. Sci. 102, 3221 (2006).

[10] P.J. Schultz, K.G. Lynn, Rev. Mod. Phys. 60, 701 (1988).

[11] F. Zheng, C.Q. He, P.F. Fang, J.G. Wang, B.Y. Xiong, K. Wang, F.W. Liu, X.Y. Peng, X.G. Xu, Z.H. Xu, S.J. Wang, Appl. Surf. Sci. 283, 327 (2013).

[12] T. Suzuki, C. He, V. Shantarovich, K. Kondo, E. Hamada, M. Matso, L. Ma, Y. Ito, $R a-$ diat. Phys. Chem. 66, 161 (2003).

[13] T. Suzuki, C. He, K. Kondo, V. Shantarovich, Y. Ito, 10.1016/s0969-806x(03)00214-7 68, 489 (2003).

[14] C. He, V.P. Shantarovich, T. Suzuki, S.V. Stepanov, R. Suzuki, M. Matsuo, J. Chem. Phys. 122, 214907 (2005).

[15] C. Bockenheimer, D. Fata, W. Possart, J. Appl. Polym. Sci. 91, 361 (2004).

[16] A. Cherdoud-Chihani, M. Mouzali, M. Abadie, J. Appl. Polym. Sci. 69, 1167 (1998).

[17] B. Mailhot, S. Morlat-Thérias, M. Ouahioune, J.-L. Gardette, Macromol. Chem. Phys. 206, 575 (2005). 